Back pain

\section{Back pain and systemic compromise}

\author{
A A Dyer, K Ashkan, J Norris
}

Answers on $p 377$.

A 30 year old white man presented to his local hospital with a four day history of lower back pain and leg weakness after a minor fall. His past medical history included a lumbar microdiscectomy eight years before. He was not diabetic and there were no risk factors for immunosuppression. Initial examination showed normal power and reflexes in both legs but there was decreased sensation on the inner aspect of the left calf, scrotum, and perianal area with normal continence. While awaiting investigation he developed an absent left ankle reflex. Magnetic resonance imaging (MRI) of the lumbar spine was then performed (see fig 1 ), on the basis of which he was referred to the regional neurosurgical unit.

On arrival, the patient was unwell, markedly tachycardic at rest, and dehydrated with severe tenderness in the lumbar spine. Neurological examination demonstrated severe weakness in both lower limbs, predominantly on the right. Plantars were equivocal and reflexes were absent throughout. Patchy sensory loss in both legs was noted which extended up to the inguinal regions, as well as saddle and perianal anaesthesia. He also had reduced anal tone, was in acute urinary retention, and required catheterisation.

\section{QUESTIONS}

(1) What is the most likely clinical diagnosis?

(2) What does the MRI scan show?

(3) Discuss the management of this condition.

Postgrad Med J 2002;78:373

\section{Authors' affiliations}

A A Dyer, K Ashkan, J Norris, Department of Neurosurgery, Hurstwood Park Neurological Centre, Haywards Heath, West Sussex, UK

Correspondence: Mr K Ashkan, Department of Neurosurgery, Level 3, Southwood Building, Great Ormond Street Hospital for Children NHS Trust, Great Ormond Street, London WCIN 3JH, UK

Submitted 30 November 2001

Accepted 24 January 2002

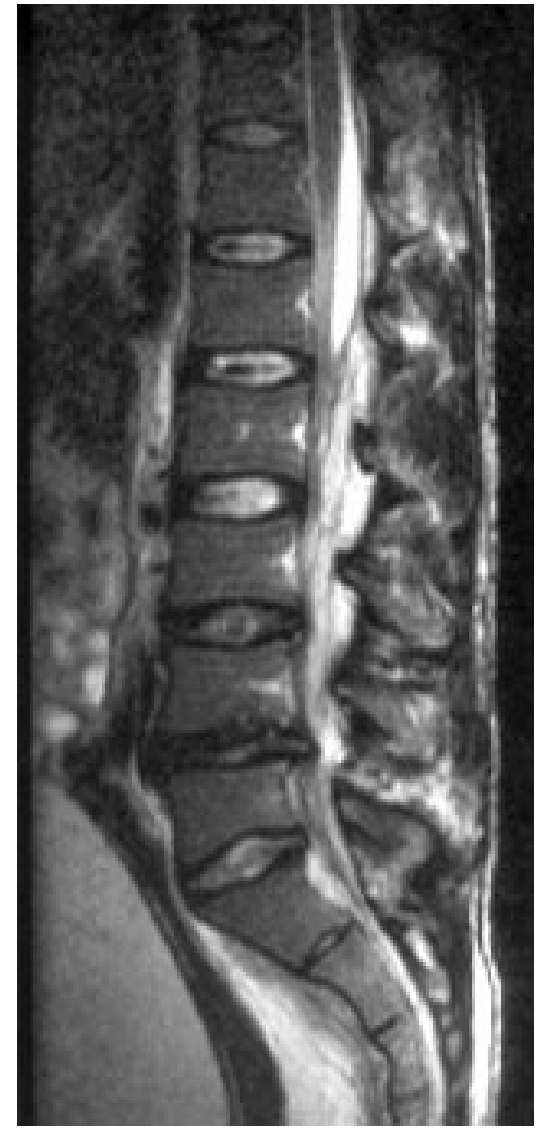

Figure 1 T2 weighted sagittal MRI scan of the lumbar spine.

Investigations at this stage included measurement of haemoglobin (202 g/l), white cell count $\left(12.2 \times 10^{9} / 1\right)$, erythrocyte sedimentation rate ( $10 \mathrm{~mm} /$ hour), urea ( $10.1 \mathrm{mmol} / \mathrm{l})$, potassium (4.6 mmol/l), creatinine $(78 \mathrm{\mu mol} / \mathrm{l})$, and calcium (2.06 mmol/l). Chest radiography was normal.

\section{QUESTIONS}

A 42 year old right handed joiner presented with a five day history of cough, fever, and aching muscles. He had previously enjoyed good health. Three days into his current illness he had attended his general practitioner and been prescribed ibuprofen, aspirin, and a "cold remedy". He had no history of recent overseas travel and other family members were well.

Over the 48 hours before hospital admission he had become much weaker and was eventually unable to walk unsupported. He was admitted to his local hospital for investigation and treatment.

At this time he complained of muscle tenderness and he found it uncomfortable to move his limbs. He had tingling in his right hand. He had also noticed that his urine had darkened in colour and his wife had noticed that his face had become swollen.

On examination he was alert, orientated, and apyrexic. There was facial oedema and all four limbs were also swollen, with marked muscle tenderness. The peripheries were cold and the urine was indeed dark brown. Neurologically, there was modest, but significant, weakness of finger abduction bilaterally (right: 4/5; left: $3 / 5$ ) as well as proximal weakness in both legs (hip flexion: 4/5 bilaterally). Passive flexion at the knees was difficult due to pain. There was no objective sensory deficit and both plantar responses were flexor.
(1) What is the most likely diagnosis and how would you confirm it?

(2) What is the possible aetiology of this condition?

(3) What potential complications may occur?

(4) How would you manage this patient?

Postgrad Med J 2002;78:373

Correspondence to: Dr Gavin McDonnell, Department of Neurology Ward 21, Quin House, Royal Victoria Hospital, Belfast, Co Antrim, Northern Ireland, BT12 6BA, UK; gvmcdonnell@doctors.org.uk

Submitted 22 May 2001

Accepted 27 July 2001 
Swollen left shoulder

\section{A case of acute swelling of the left shoulder}

\section{S Parekh, M Pisaneschi, W Sequeira}

elbow with her right hand. The left shoulder had a large effusion with diffuse swelling of the arm but no overlying redness or tenderness. Movement of the joint was uncomfortable and reduced. All other joints in the arm were normal. Cytology of the $150 \mathrm{ml}$ of bloody fluid aspirated from her shoulder identified fragments of bone and cartilage. There were no crystals and routine bacterial and mycobacterial cultures were negative. She had a positive PPD (purified protein derivative).

A repeat radiograph of the left shoulder done six weeks later (see fig 1) prompted a more detailed neurological examination. This revealed reduced pain and temperature over the left shoulder and upper arm extending towards the midline with reduced deep tendon reflexes compared to the opposite limb.

A magnetic resonance image (MRI) of the cervical spine was done to confirm the diagnosis (see fig 2).

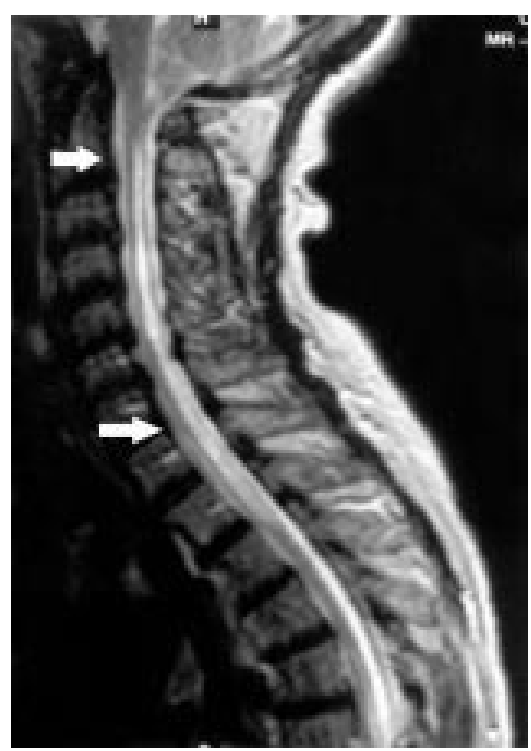

Figure 1 Radiograph of the left shoulder done six weeks after presentation; this demonstrates sharp margins almost as if part of the head of the humerus was surgically excised.

done to exclude a deep venous thromboof her left upper extremity normal. An incidental finding of a subluxed left shoulder on chest radiography prompted a consultation by a specialist.

The rheumatologist noticed the patient supporting her left arm at the

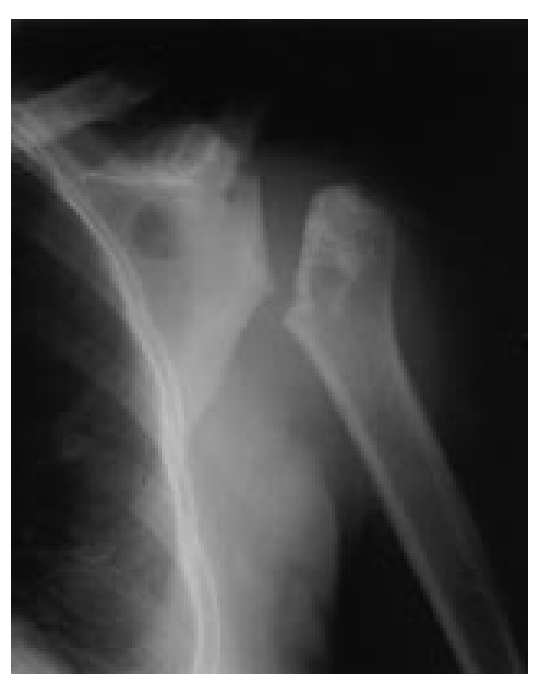

Figure 2 MRI of the cervical spine demonstrating the large syrinx between the arrows.

\section{QUESTIONS}

(1) What disease process are the $x$ ray and MRI findings diagnostic of?

(2) What are the other causes of osteolysis?

(3) What are the other clinical features of this disease?

Postgrad Med J 2002;78:374

S Parekh, W Sequeira, Department of Medical College, Chicago, Illinois, USA

M Pisaneschi, Department of Radiology

Correspondence to: Dr Winston Sequeira, Division of Rheumatology, Cook County Hospital and Rush Medical College, 1900 W Polk \#650, Chicago, IL 60612, USA; Wsequeir@rush.edu

Submitted 3 September 2001

Accepted 19 October 2001

\section{Authors' affiliations} Medicine, Cook County Hospital and Rush 
Abdominal pain

\section{Abdominal pain in a diabetic myeloma patient with cirrhosis C Gönen, B Öksüzoglu, S Yalçin}

Answers on $p 379$.

$\Lambda$ 57 year old woman with cirrhosis was admitted to the emergency room because of hepatic encephalopathy. She had a 10 year history of multiple myeloma but she tolerated chemotherapy poorly and had required plasmapheresis twice in the past year. In her last evaluation three months previously her IgG concentration was 146.0 $\mathrm{g} / \mathrm{l}$ (reference range 8.0-18.0 g/l) with low concentrations of IgM and IgA and a monoclonal spike on serum protein electrophoresis. Because her glucose levels were in the range of 15 to $21 \mathrm{mmol} / \mathrm{l}$

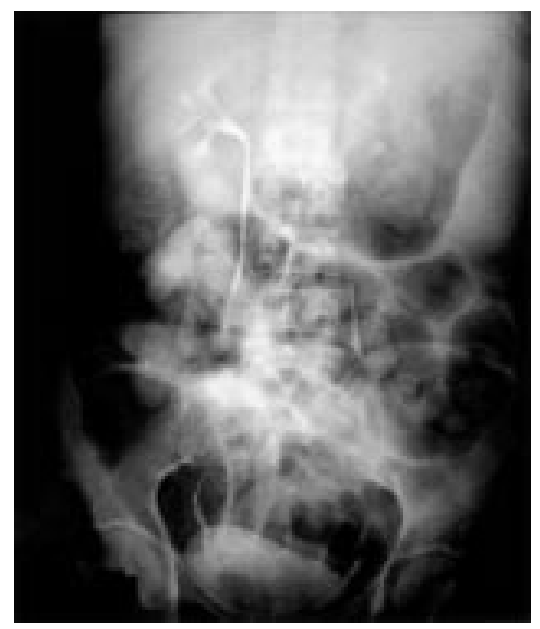

Figure 1 Plain abdominal film of the patient.
(4.2-6.6 mmol/l), insulin treatment was initiated

One week after hospitalisation, despite appropriate treatment for hepatic encephalopathy and some improvement, her condition worsened again. She was unresponsive to verbal commands. On physical examination lower abdominal tenderness was present without any rebound. Because the patient has no detectable ascites, paracentesis was not performed. Laboratory findings at this point were as follows: total protein $85 \mathrm{~g} / \mathrm{l}$ (60-78 g/l), albumin $22 \mathrm{~g} / \mathrm{l}(32-48 \mathrm{~g} / \mathrm{l})$ total bilirubin $67.6 \mu \mathrm{mol} / \mathrm{l}(2-21 \mu \mathrm{mol} / \mathrm{l})$, creatinine $16.7 \mu \mathrm{mol} / \mathrm{l}(50-100 \mu \mathrm{mol} / \mathrm{l})$, glucose $7.32 \mathrm{mmol} / \mathrm{l}(4.2-6.6 \mathrm{mmol} / \mathrm{l})$, international normalised ratio 1.81 (0.75-1.5), alanine aminotransferase $1.92 \mu \mathrm{kat} / \mathrm{l}(0.1-0.8 \mu \mathrm{kat} / \mathrm{l})$, and aspartate aminotransferase $0.58 \mu \mathrm{kat} / \mathrm{l}$ (0.16-0.66 $\mu \mathrm{kat} / \mathrm{l})$. Bladder catheterisation yielded

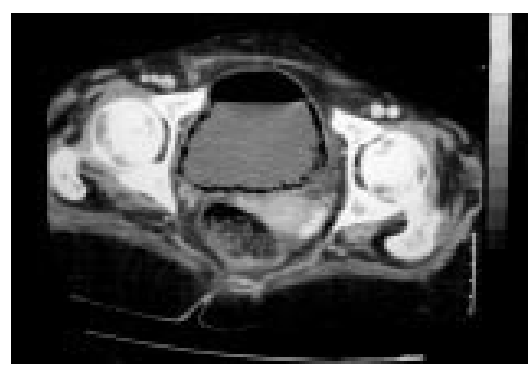

Figure 2 Computed tomography of the abdomen at the level of bladder. foul smelling and red coloured urine. Analysis revealed marked pyuria (90 white blood cells/high power field) and haematuria (1927 red blood cells/high power field).

In order to identify the cause of abdominal pain in this diabetic myeloma patient with Child C cirrhosis, abdominal computed tomography and direct $x$ ray abdominal radiography were carried out (see figs 1 and 2).

Radiological studies revealed gas within the bladder wall and a urinary tract infection was suspected. After obtaining a urine culture, empiric antibiotic therapy with piperacillin $4 \mathrm{~g}$ plus tazobactam $0.5 \mathrm{~g}$ four times a day was initiated. A bladder catheter was left in place and blood glucose levels were strictly regulated.

Urine culture subsequently revealed Proteus vulgaris. Three days later a control urine culture was negative and abdominal pain improved.

\section{QUESTIONS}

(1) What is the differential diagnosis?

(2) What abnormalities are seen on the radiographs?

(3) What are the predisposing factors?

(4) How would you treat this condition?

Postgrad Med J 2002;78:375

\section{Authors' affiliations}

C Gönen, Hacettepe University, Department of Internal Medicine, Ankara, Turkey B Öksüzoglu, S Yalçin, Hacettepe University, Institute of Oncology, Medical Oncology Department, Samanpazari, 06100, Ankara, Turkey

Correspondence to: Dr Öksüzoglu; bernaoksuzoglu@hotmail.com

Submitted 15 May 2001

Accepted 19 December 2001 
prevented him from sitting him up in

\section{An eponymous reaction to a knife wound \\ D Blackburn, D J Werring, S E J Connor, N Munro, N P S Bajaj}

Answers on $p 379$.

A 27 year old man was admitted to casualty having been stabbed in the back at a nightclub. He had taken an ecstasy tablet and had consumed some alcohol. On assessment, he was alert and cooperative with a $2 \mathrm{~cm}$ long, visible knife wound on the right hand side of the thoracic vertebral column. Examination of the cardiovascular, respiratory, and gastrointestinal systems was normal.

Neurological examination revealed no abnormality of cranial nerves or upper limbs. Lower limb examination revealed normal right leg tone, power, and reflexes. Left leg tone was spastic with clonus and pyramidal weakness. The left knee and ankle jerks were pathologically brisk and he had an up-going left plantar. The right plantar was flexor. Light touch was normal on the right and abnormal on his left leg up to a soft level at D8. He could not detect temperature or pinprick in his right leg up to Dlo. Vibration sense was absent bilaterally up to the anterior superior iliac spines. Joint position sense was absent bilaterally at the toes and the ankles. The left lower abdominal reflex was absent with all other abdominal reflexes being normal. Anal tone was normal. The next day he developed a severe frontal headache that bed. There was accompanying nausea without visual aura or photophobia.

\section{QUESTIONS}

(1) Where is the anatomical site of injury and which spinal tracts have been damaged?

(2) What is the name of this syndrome?

(3) Give three other causes of this syndrome.

(4) What is the cause of his headache?

(5) How would you treat his headache if the symptoms persisted?

Postgrad Med J 2002;78:376

\section{Authors' affiliations}

D Blackburn, D J Werring, N Munro, N P S Bajaj, Department of Neurology, King's College Hospital, London, UK

S E J Connor, Department of Neuroradiology

Correspondence to: Dr N P S Bajaj, Department of Neurology, King's College Hospital, Mapother House, De Crespigny Park, Camberwell, London SE5 8AF, UK; spgtnpb@iop.kcl.ac.uk

Submitted 28 August 2001

Accepted 4 January 2002 